\title{
Time- and Position-Dependent Modeling of High-Power Low-Repetition-Rate Er-Yb-Fiber Amplifier
}

\author{
I. Pavlov ${ }^{1}$, E. Dülgergil ${ }^{2}$, P. Elahi ${ }^{1}$ and F. Ö. Ilday ${ }^{1}$ \\ ${ }^{1}$ Department of Physics, Bilkent University, 06800 Ankara, Turkey \\ ${ }^{2}$ Meteksan Savunma, A. Ş., 06800, Ankara, Turkey
}

There is rapid progress in the development of high-power fiber lasers due to their robust operation, low cost, high beam quality at high powers. There are various applications, such as laser sensing, LIDAR applications, processing of specific materials, which require robust and high-power pulsed laser sources at $1550 \mathrm{~nm}$ with high beam quality. Achievement of high peak power with low repetition rate is challenging due to well-known problems of strong nonlinear effects and amplified spontaneous emission (ASE) build-up between pulses. In order to reach highest efficiency, the design of each stage of amplification should be carefully optimized. Numerical modeling can be a great tool due to the large number of parameters involved [1]. To date, most modeling efforts of fiber amplification have assumed either a lumped gain model for pulse propagation or a distributed, position-dependent gain model for CW signal for computational simplicity. Here, we investigate both time- and position-dependent gain dynamics numerically, which are used to optimize experimental results.
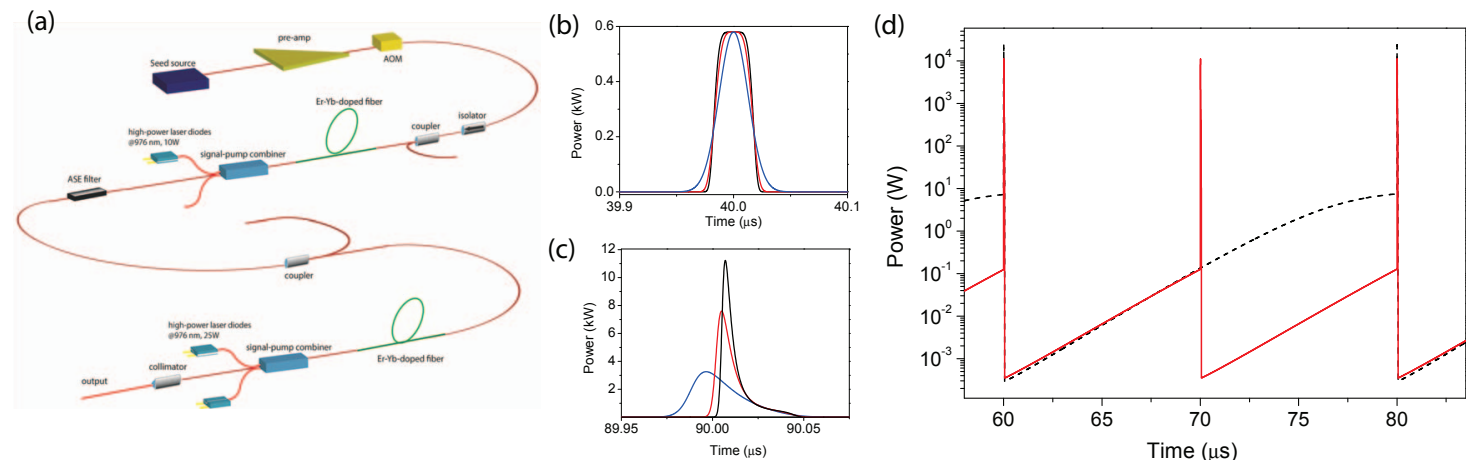

Fig. 1 (a) The schematic of experimental setup. (b) Different pulse shapes with same peak power and pulse energy used as seed for investigation of dynamic gain saturation in the final stage amplifier. (c) Simulation results for the final stage amplifier for pulse shapes shown in (c). Note that the leading edge of the seed pulse shape strongly influences pulse shape after amplification. (d) Simulation results for ASE generation at $50 \mathrm{kHz}$ (black curve) and 100 (red curve) kHz repetition rates.

We solve rate equations for Er-doped fiber (three-level system) and Er-Yb-co-doped fiber (six-level system) using finite differences method. The fiber is divided into small segments and for each segment, the rate equations are solved for both forward and backward propagating signals for a fixed time interval. Next, the time step is increased, and the process is iterated. This scheme allows us to analyze pulse propagation with population levels dependent both on time and position along the fiber. This treatment is evidently necessary to correctly model processes such as dynamic gain saturation and ASE creation, since the physical length of a few-ns pulse is comparable to the length of a typical gain fiber. Simulation results were confirmed by experiments, based on which the experimental setup was iteratively optimized. The experimental setup is shown in Fig. 1(a). It consists of a seed source, which is a current-modulated diode laser, a core-pumped Er-fiber preamplifier, an acousto-optic modulator to reduce repetition rate to $50 \mathrm{kHz}$ or $100 \mathrm{kHz}$ and a three-stage amplifier system. The first stage comprises of a single-clad Er-doped fiber and the last two stages are based on cladding-pumped Er-Yb co-doped fiber with 10$\mu \mathrm{m}$ and $12-\mu \mathrm{m}$ core diameter. Following the final amplifier, pulses traverse standard singlemode fiber (SMF-28) to clean up any residual high-order modes before being extracted from fiber. In simulations, the seed source is modeled with Gaussian pulses with peak and average power of $50 \mathrm{~mW}$ and $0.3 \mathrm{~mW}$, respectively, corresponding to our experimental values. Simulation results confirm that sufficient seed power is needed from each stage to the next for its saturation and prevention of ASE creation. However, even at high pulse energy and average power after the middle stage ( $1.2 \mathrm{~W}$ in simulation and experiment), at $50 \mathrm{kHz}$ repetition rate we observe significant amount of ASE creation between pulses (see Fig. 1 (d)). By comparison, at $100 \mathrm{kHz}$, the ASE generation was negligible.

In conclusion, we report on time- and space-dependent modeling of amplification of nanosecond pulses in a multi-stage fiber amplifier with good agreement to experiments. The influence of seed pulse shape on amplified pulse shape, and ASE creation are investigated. Guided by numerical simulation, we optimized our setup and generated 10-W, 5-ns, 100-kHz pulses from an all-fiber, diffraction-limited and ASE-free fiber amplifier.

\section{References}

[1] E. Lim, S. Alam, and D. J. Richardson, "High-energy, in-band pumped erbium doped fiber amplifiers", Opt. Express 20, 18803 (2012). 